\title{
Higher order effective interactions and effective bosonized model for 2-N particle states
}

\author{
Fabio L. Braghin,a \\ ${ }^{1}$ Instituto de Física, Univ. Fed. de Goiás, P.B.131, Campus II, 74.001-970, Goiânia, GO, Brazil
}

\begin{abstract}
In this work, an effective fermion model with particular higher order interactions given by: $I_{I I}=\sum_{n}^{N} g_{2^{n}}\left(\bar{\psi}_{a} \psi_{a}\right)^{2^{n}}$, for finite $N$, is investigated by means of the auxiliary field method by taking into account $2 n$-particle states as proposed in Ref. [1]. The bosonized model was found to exhibit an approximate symmetry when expanded for weak field fluctuations around the mean field solutions. In the present work, the role of the mean field solutions for the corresponding auxiliary fields is investigated. With the integration of fermions, the resulting determinant is expanded in a polynomial boson model in the weak field approximation and the approximate symmetry found for this series does not appear if the boson mean fields are considered to be zero.
\end{abstract}

\section{Introduction}

The auxiliary field method [2-5] is a powerful non perturbative method which reduces the non linearities of a theory at the expenses of introducing interacting auxiliary fields (a.f.). It has been successefully applied in different systems from quantum field, strong interacting and nuclear systems models to many body atomic and electronic systems [5-9]. N-body effective interactions can contribute in few and many body systems and it is one extremely difficult problem to take them into account. Lately it has been shown that these higher order interactions constitute important part of the Effective Field Theory approach (EFT) [10,11]. Some new few body states and interactions have been observed in several systems in the last years such as in meson spectroscopy with the appearance of tetraquark states and in cold atoms. A particularly interesting observed feature is an apparent approximate degeneracy of 2-particle and 4-particle states in light scalar mesons (masses below/around $1 \mathrm{GeV}$ ) [12] and also in 2 and 3 or 4 particle states in cold atoms by means of their binding energies [13]. Even though this last systems are non relativistic one might search for general feature(s) of few body states built with few body interactions. In the present work a complementary calculation to Ref. [1] is performed based in a dynamical fermion effective model. In the present work, the particular series addressed in [1] given by $I_{I I}=\sum_{n}^{N} g_{2^{n}}\left(\bar{\psi}_{a} \psi_{a}\right)^{2^{n}}$, for finite $N$, is analysed within the auxiliary field method if there are no mean field solutions. The logics of the work is the following. A dynamical model with higher order interactions is considered and it is bosonized by introducing a set of $\mathrm{N}$ auxiliary fields. Although from EFT power counting or renormalization group arguments the more relevant terms of a series of interactions are not those present in the series $I_{I I}$, the idea of the present development is the following: to investigate the role of separate terms or classes of higher order effective interactions by switching

ae-mail: braghin@ufg.br 
on and off the others to understand their roles in the bosonized resulting effective model, which describes sets of $2 n-$ particles composite states. The minimum number of a.f. is introduced such as to cancel out all the interacting terms by means of minimum number of shifts, which are considered to be the simplest ones. Although this approach is strictly valid for weak fluctuations of the a.f. with respect to a coupling constant scale, it was shown in Ref. [1] that it yields the correct expressions if this weak field approximation is lifted. By integrating out the original fermion fields a bosonized model for a collection of $\mathrm{N}$-particle states is obtained which can be expanded in polynomial boson effective interactions. The saddle point equations of the bosonized model are considered to have only trivial solutions without the formation of mean fields differently from Ref. [1]. The resulting polynomial effective model is found and compared to the case in which there is non trivial a.f. mean field (condensates). It will be shown that the new approximate symmetry between $\mathrm{N}$-fermion states appear only if the condensates (mean fields) are formed, i.e. if mean fields are taken into account.

\section{Auxiliary fields and bosonization}

Consider the following generating funcional with a polynomial effective model:

$$
Z=\int \mathcal{D}\left[\bar{\psi}_{a}, \psi_{a}\right] e^{i \int_{x} \mathcal{L}_{I I}\left[\psi_{a}, \bar{\psi}_{a}\right]}
$$

where the following series of bilinears will be considered:

$$
\mathcal{L}_{I I}=\bar{\psi}_{a}(x)\left(i \not \partial-m_{a}\right) \psi_{a}(x)+\sum_{n=1}^{N} g_{2^{n}}\left(\bar{\psi}_{a} \Gamma \psi_{a}\right)^{2^{n}},
$$

where $N$ even and $\Gamma=I$ will be addressed; where $g_{2^{n}}$ are the effective coupling constants with dimension: $\left[g^{2^{n}}\right]=M^{d-(d-1) 2^{n}}$, where $M$ has dimension of mass, $m_{a}$ are the masses for each of the fermion species and the index $a=1 \ldots N_{r}$ stands for the fermion components. The auxiliary fields, with dimension $\left[M^{2}\right]$, are introduce by means of $N / 2$ unity integrals that are given by:

$$
N^{\prime} \int \mathcal{D}\left[\varphi_{n}\right] e^{-i \int_{x} \frac{1}{2} \sum_{n}^{N} \frac{1}{d_{n}} \varphi_{n}^{2}(x)}=1
$$

The simplest necessary shifts of the auxiliary fields that cancel out the interactions can be written as:

$$
\varphi_{n}^{2} \rightarrow\left(\varphi_{n}-\beta_{n}\left(\bar{\psi}_{a} \psi_{a}\right)^{2^{n-1}}\right)^{2}
$$

where $\beta_{n}$ are dimensionful parameters that are determined by imposing the corresponding cancelations of all polynomial interactions. There are other possible shifts in the auxiliary fields, however these are the simplest ones with the minimal number of auxiliary fields that cancel out all the polynomial interactions without introducing further previously non existing interations. This reduces eventual ambiguities in the procedure.

For an arbitrary $n$, these conditions can be written in the following form:

$$
\begin{array}{llrl}
g_{2^{n}}=-\frac{\beta_{n+1}}{d_{n+1}} \varphi_{n+1}+\frac{\beta_{n}^{2}}{2 d_{n}}, & \text { for } \mathrm{n}<\mathrm{N}, \\
g_{2^{n}}=\frac{\beta_{n}^{2}}{2 d_{n}}, & \text { for } \mathrm{n}=\mathrm{N} .
\end{array}
$$

From these expressions the parameters $\beta_{n}$ might become functions of the fields $\varphi_{n+1}$ without changing the unity Jacobian. This minimal procedure is valid when all the fermion coupling constants, except 
$g_{2^{N}}$, are quite strong and the subset of $\varphi_{N-1}$ fields only assume positive values and/or these auxiliary fields are weak with respect to the mean field which are weaker than the (normalized) fermion coupling constants. This means that higher order auxiliary fields, which are introduced to cancel out progressively more irrelevant fermion interactions, are progressively weaker, i.e. $\left|\varphi_{m} \beta_{m}\right|<g_{2^{m-1}}$ for positive coupling constants.

The resulting effective action for fermions interacting with the auxiliary fields is given by:

$$
S_{e f f}=\int d^{4} x\left[\bar{\psi}_{a}\left(i \not \supset-m_{a}+\frac{\beta_{1}}{d_{1}} \varphi_{1}\right) \psi_{a}-\sum_{n=1}^{N} \frac{1}{2 d_{n}} \varphi_{n}^{2}\right] .
$$

The saddle point equations for these auxiliary fields provide relations between the ground state average of the auxiliary fields the progressively large power of bilinears, i.e. $\varphi_{n} \sim\left(\bar{\psi}_{a} \psi_{a}\right)^{n}$, with an implicit summation on the index $a$. Therefore the auxiliary fields represent corresponding scalar composite fermion states, basically as a sum of states. By integrating out fermions and by using that $\operatorname{det} A=$ $\exp (\operatorname{Tr} \ln A)$ it yields:

$$
S_{e f f}=-i \operatorname{Tr} \log \left(i \not \partial-m_{a}+\frac{\beta_{1}}{d_{1}} \varphi_{1}\right)-\sum_{n=1}^{N} \int_{x} \frac{1}{2 d_{n}} \varphi_{n}^{2},
$$

where $\operatorname{Tr}$ is the traces taken over all the internal indices and integration over space-time. According to expression (5), there is a recursive dependence on all the a.f. in the determinant because: $\beta_{1}\left[\varphi_{n}\right]$. Only the trivial zero solutions for the gap equation will be considered.

\section{Expanded effective boson model}

The first terms expansion corresponds to:

$$
S_{e f f} \simeq S_{e f f,(0)}\left[\varphi_{i}^{(0)}\right]+\left.\sum_{i}^{N} \sum_{j}^{N} \frac{1}{n_{i} ! n_{j} !} \int_{x_{1}, x_{2}} \frac{\delta^{2} S_{e f f}}{\delta \varphi_{i}\left(x_{1}\right) \delta \varphi_{j}\left(x_{2}\right)}\right|_{\varphi_{i}=\varphi_{i}^{(0)}} \varphi_{i}\left(x_{1}\right) \varphi_{j}\left(x_{2}\right)+\text { h.o. }
$$

where $\int_{x_{1}, x_{2}}=\int d x_{1} \int d x_{2}$, h.o. stands for (even) higher order derivatives, $n_{i}, n_{j}$ in the second derivative term and $n_{i}, n_{j}, n_{k}$ in the third order are such that $n_{i}+n_{j}=2$ and $n_{i}+n_{j}+n_{k}=3$ respectively. The first derivative term is set to zero due to the stability condition.

By assembling the interaction terms, it yields for the first three auxiliary fields:

$$
\mathcal{V}_{I}^{\text {eff }}=\sum_{j=2} \frac{c_{j}}{2} \varphi_{1}^{j}+\sum_{j, k=1} t_{j, k} \varphi_{1}^{j} \varphi_{2}^{k}+\sum_{j, k, i=1} t_{j, k, i} \varphi_{1}^{j} \varphi_{2}^{k} \varphi_{3}^{i}
$$

where $c_{j}$ are the resulting self interaction coupling constants for $\varphi_{1}, t_{j, k}$ for $j+k \geq 2$ and $t_{j, k, i}$ for $j+k+i \geq 3$. It is seen that only the first a.f. develops a mass term, and higher order fields only couple to successively lower order a.f., i.e. $\varphi_{n}$ only develops couplings to all the a.f. of the type $\left(\prod_{m}^{n-1} \varphi_{m} \varphi_{n}\right)$. This is a result of the zero condensates case. For the sake of comparison the following expression obtained in Ref. [1] is reproduced here for the case of non zero condensates in the case the fields are suitably redefined to incorporate:

$$
\begin{aligned}
\mathcal{V}_{I}^{e f f}= & \frac{1}{2}\left[c_{2} \chi_{1}^{2}+\left(c_{2}+c_{2,2}\right) \chi_{2}^{2}+\left(c_{2}+c_{2,2}+c_{2,3}\right) \chi_{3}^{2}+\left(c_{2}+c_{2,2}+c_{2,3}+c_{2,4}\right) \chi_{4}^{2}+\ldots\right] \\
& +\sum_{n \geq 3}\left[c_{n} \chi_{1}^{n}+\left(c_{n}+c_{n, 2}\right) \chi_{2}^{n}+\left(c_{n}+c_{n, 2}+c_{n, 3}\right) \chi_{3}^{n}+\ldots\right]+\sum_{i, j, k} t_{i, j, k} \chi_{1}^{i} \chi_{2}^{j} \chi_{3}^{k}, \\
& t_{i, j, k} \text { for } i+j+k \geq 2, \text { at least two indices non zero, }
\end{aligned}
$$


where $c_{n}$ and $c_{n, m}$ are the resulting self interaction coupling constants and contributions for masses, and also the couplings $t_{i, j, k}$ are those couplings between at least two different components, being that at least two indices are non zero, i.e. $i, j \neq 0$ or $i, k \neq 0$ and so on. From this expression the limit of progressively relatively large condensates yielded an effective potential with an approximate symmetry. By comparing expressions (9) and (10) it is seen there is a complete absence of the explicit effective and approximate symmetry that was found in the case of non zero (relatively large) higher order condensates [1].

\section{Conclusions}

In this work an effective bosonized model for $n$-fermion scalar states was built from a dynamical effective fermion model with very particular higher order interactions by means of the auxiliary field method in the case of zero mean fields. By integrating out fermions the resulting determinant was expanded in polynomial boson effective interactions. The approximate symmetry found in Ref. [1] in the case of relatively large higher order condensates (mean fields) was found to be completely absent in the case of zero scalar fields condensates. The resulting effective potential (9) exhibits a preferential role for the lowest order scalar a.f. $\left(\varphi_{1}\right)$ which appears in all interaction terms.

\section{Acknowledgements}

The author thanks short discussion with P. Bedaque, and financial support from FAPEG-Goias and CAPES-MCT and CNPq, Brazil.

\section{References}

[1] F.L. Braghin, submitted to publication, arXiv:1505.01209 [hep-th].

[2] T. Kashiwa, Phys. Rev. D 59, 085002 (1999).

[3] D.J. Gross, A. Neveu, Phys. Rev. D 10, 3235 (1974).

[4] H. Kleinert, Electronic Journal of Theoretical Physics 8, 57 (2011).

[5] J.W. Negele, H. Orland, Quantum Many-Particle Systems, Addison-Wesley, 1988.

[6] S.P. Klevansky, Rev. of Mod. Phys. 64, 649 (1992).

[7] E. Baum, Nucl. Phys. B 266, 547 (1986).

[8] H. Kleinert, Lectures presented at the Erice Summer Institute 1976, in Understanding the Fundamental Constituents of Matter, Plenum Press, New York, ed. by A. Zichichi, 289 (1978).

[9] F.L. Braghin, F.S. Navarra, Phys. Rev. D 91, 074008 (2015).

[10] P.F. Bedaque, U. van Kolck, Annu. Rev. Nucl. Part. Sci. 52, 339 (2002).

[11] E. Braaten, H.-W. Hammer, Phys. Rept. 428, 259 (2006).

[12] For example in: K.A. Olive et al. (Particle Data Group), Chin. Phys. C 38, 090001 (2014). G. 't Hooft, G. Isidori, L. Maiani, A.D. Polosa, V. Riquer, Phys.Lett.B 662, 424 (2008).

[13] For example in: J. H. Gurian, P. Cheinet, P. Huillery, A. Fioretti, J. Zhao, P. L. Gould, D. Comparat, and P. Pillet Phys. Rev. Lett. 108, 023005 (2012). F. Ferlaino, S Knoop, M. Berninger, W. Harm, J. P. D’Incao, H.-C. Nagerl, R. Grimm Phys. Rev. Lett. 102, 140401 (2009). 